\title{
Note on Vertical Correlations of the Wind
}

\author{
C. E. Buell \\ Kaman Nuclear, Colorado Springs, Colo. \\ 10 June 1961
}

Regarding the questions raised by Dr. A. Kochanski on page 159 of the April 1961 issue of the Journal of Meteorology, it is felt that the answers to some of these questions can be suggested at the present time. The sections following propose answers to the corresponding three questions raised.

(1) The mid-tropospheric maximum of $R$ is intimately connected with the "depth" of wind systems. The curvature of the wind correlation function near the value of zero height difference may be interpreted in precisely these terms. In rather idealized terms, one may write

$$
R=1-H^{2} / 2 \lambda_{d}{ }^{2}+\cdots
$$

in which $\lambda_{d}$ might be called a "depth" measure, and where $H$ is the height difference. In actuality, this expression must be modified drastically to account for the effects of observational error and small scale wind perturbations. This form of depth parameter is similar to that proposed for height systems or pressure systems (Buell, 1954).

One should note in passing that the value $R=\left(r_{u}+r_{v}\right) / 2$ is more closely related to the simple stretch vector correlations (Buell, 1960) than to either the combination stretch-and-turn or the total vector correlation.

The larger values of the "depth" of wind systems in the midtroposphere are expected since this is the middle of a zone of rather homogeneous structure in the vertical. The correlations at the lower levels are expected to fall off very rapidly due to the effects of "surface" systems whose structure tends to merge with that of the mid-troposphere systems several kilometers above sea level. Similarly, the wind systems in the lower stratosphere tend to be "shallow," a fact at least partially associated with the low correlation between height and temperature at a point there; high correlation being necessary for deep systems.

It should be emphasized that in viewing wind correlations in one (vertical) dimension, only a small part of the picture is displayed. The treatment of the relation between wind correlation and height correlation given elsewhere (Buell, 1960) can be nicely applied to three dimensions. One considers the wind correlation tensor formed from winds at $P(x, y, z)$ and at $P^{\prime}(x+\xi$, $y+\eta, z+\zeta)$. For fixed $x, y, z$ and $\zeta$, one may plot contours of the components of the wind correlation tensor, the values of $r_{u}=r_{11}$ and $r_{v}=r_{22}$ being the most important. These contours are very similar to those of Fig. 3 in Buell (1960), $(\zeta=0)$, even when $\zeta \neq 0$. There are some important differences: (a) the value of maximum correlation is now at a value less than 1 ; (b) the center of maximum correlation shifts from the location of $P$ (westward for $\zeta>0$, the eastward for $\zeta<0)$. This, of course, corresponds to the well-known tilt of height systems. When considering correlations in the vertical $(\xi=\eta=0)$ there are then two effects that become confounded, the decay of the maximum correlation and the tilt of the systems. This is evidenced by noticing the detailed differences between $r_{u}$ and $r_{v}$ for $\zeta \neq 0$. Generally $r_{u}>r_{v}, \zeta \neq 0$, since $r_{v}$ falls off more rapidly with distance in the $\mathrm{E}-\mathrm{W}$ direction than does $r_{u}$, this also being the direction of tilt of the systems.

(2) The variations between the decay curves of Fig. 6 (Kochanski, 1961) may be tentatively interpreted as being due to the mixture of wind systems of different characteristics. One notes that the "hump" in the 700-, 500-, and 400-mb level curves appears at approxi- 
mately the 250 -mb level (i.e., at a separation corresponding to the height difference between $250 \mathrm{mb}$ and the other levels enumerated). [One might imagine a slight hump at this point on the $850-\mathrm{mb}$ curve and on the $300-\mathrm{mb}$ curve between the first point $(250 \mathrm{mb})$ and $\xi=H=0$.] This would suggest that the variation in shape is tropopause connected. However, since the curves are averages, the tropopause position would of necessity be badly defined. The curves for the 200 -, 150-, and 100-mb levels contain only points attributed to stratosphere conditions and parallel each other with the least separation of the group shown.

The correlation coefficient decay curves are intimately connected with the physical processes taking place. It is to be expected that different curves would be encountered in high and low latitudes (with a transition zone), and in lower and upper troposphere and lower stratosphere.

(3) In casting about for a "predictor" for $R$, it might be suggested that since the geostrophic wind is such a large fraction of the real wind and since the geostrophic wind is a function of the height field only, the height correlations may well be intimately connected with $R$. When a covariance is "factored" into the standard deviations and the correlation coefficient, the factors each have separate significance : the standard deviations describing the inherent variability of the system, the correlation describing the internal "connectedness" of the system (size, process, etc.). The approximate relation between the wind variability and height variability is well known (Buell, 1957). The basic equations relating the components of the wind correlation tensor to the height correlations are also well known (Buell, 1960). One would expect that this physical relation would lead to an intimate connection between $R$ and the height correlation in the vertical (a quantity somewhat easier to get at than $R$ ). Little direct physical connection would be expected between a correlation measure $(R)$ and a variability measure (standard deviation).

Dr. Kochanski's detailed synthesis of so many highly varied wind correlations into a reasonably well-ordered system should be of great usefulness for many meteorological applications.

\section{REFERENCES}

Buell, C. E., 1954: Some relations among atmospheric statistics. J. Meteor., 11, 238-244.

- $, 1957:$ An approximate relation between the variability of wind and the variability of pressure or height in the atmosphere. Bull. Amer. meteor. Soc., 38, 47-51.

- 1960: The structure of two-point wind correlations in the atmosphere. $J$. geophys. Res., 65, 3353-3366.

Kochanski, Adam, 1961: Models of vertical correlations of the wind. J. Meteor., 18, 151-159. 